\title{
Desenvolvimento de Filmes de Nananocompósitos Polipropileno/Argila Organofílica para Embalagens
}

\author{
Arthur R. A. Araújo, Wandemberg B. de Mesquita \\ Felinto Indústria e Comércio LTDA \\ Eduardo L. Canedo \\ PolyTech, Prospect, Connecticut, USA \\ Claudia M. O. Raposo \\ Unidade Acadêmica de Mineração e Geologia, UFCG \\ Daniela de L. A. C. S. Andrade, Laura H. de Carvalho, Suédina M. de L. Silva \\ Unidade Acadêmica de Engenharia de Materiais, UFCG
}

Resumo: Neste trabalho foram preparados filmes de nanocompósitos de polipropileno (PP) contendo $1 \%$ em massa de bentonita modificada organicamente com tecnologia nacional (APO). Polipropileno enxertado com anidrido maleico (PP-g-AM) e copolímero de etileno e álcool vinílico (EVOH) foram utilizados como agentes compatibilizantes. Os nanocompósitos foram preparados através da intercalação no estado fundido em extrusora de filme plano. A influência do tipo e do teor de compatibilizante e do tipo de polipropileno, na morfologia, propriedades mecânicas e de barreira ao vapor d'água dos referidos filmes foi investigada. Os dados de difração de raio X evidenciam que apesar da morfologia dos nanocompósitos ter sido afetada pelo tipo de compatibilizante, esta praticamente não variou com o teor de compatibilizante utilizado. As morfologias e as propriedades de barreira ao vapor d'água dos nanocompósitos preparados com os polipropilenos PP H401 e PP H07D-00 são semelhantes. Entretanto, aqueles preparados com PP H07D-00 apresentaram melhores propriedades mecânicas, excetuando a resistência da termossoldagem à tração, do que os preparados com a matriz polimérica PP H401, e a transparência dos nanocompósitos preparados com PP H07D-00 foi idêntica à da matriz polimérica sem argila. Os resultados indicam que os filmes de nanocompósitos PP H07D-00 compatibilizados com 15\% de PP-g-AM e contendo 1\% de argila organofílica APO podem ser promissores para aplicação em embalagens.

Palavras-chave: Filmes de nanocompósitos, argila organofílica, polipropileno, compatibilizante.

\section{Development of Polypropylene/Organoclay Nanocomposite Films for Packaging}

\begin{abstract}
This work deals with polypropylene (PP) nanocomposite films containing $1 \mathrm{wt}$. (\%) bentonite clay organically modified with national technology (APO). Maleic anhydride grafted polypropylene (PP-g-MA) and ethylene vinyl alcohol (EVOH) were used as compatibilizing agents. Nanocomposites were prepared by melt intercalation in a flat film extruder. An investigation was made of the influence from the type and content of compatibilizer and type of polypropylene on the morphology, mechanical properties and barrier properties to water vapor of these films. X-ray diffraction data show that while the nanocomposite morphology was affected by the type of compatibilizer, it was relatively insensitive to its content. The morphologies and barrier properties to water vapor of the nanocomposites prepared with the two polypropylenes (PP H401 and PP H07D-00) were similar. However, except for the heat sealing properties, those prepared with PP H07D-00 showed better mechanical properties. The transparency of P715AMAPO nanocomposite was similar to that of the P715AM matrix. The results indicated that PP H07D-00 nanocomposite films compatibilized with 15\% PP-g-AM and containing $1 \%$ APO organoclay may be promising for packaging applications.
\end{abstract}

Keywords: Nanocomposite films, organoclay, polypropylene, compatibilizer.

\section{Introdução}

Com o advento dos nanocompósitos poliméricos, as indústrias de embalagens vêm vislumbrando a possibilidade de obter produtos com boas propriedades mecânicas e de barreira a gases empregando polímeros comerciais usuais, a exemplo das poliolefinas, polipropileno (PP) e polietileno (PE), carregados com argilas organofílicas ${ }^{[1-3]}$.

Estudos sobre nanocompósitos PP/argila organofílica são amplamente divulgados na literatura ${ }^{[4-20]}$. Entretanto, o número de publicações sobre filmes de nanocompósitos PP/argila organofílica para aplicação no setor de embalagens alimentícias é limitado ${ }^{[21-23]}$ e a literatura tem mostrado que, ao contrário dos nanocompósitos preparados com matrizes polares como poliamidas, poliésteres e policarbonatos, os preparados com matrizes apolares poliolefínicas não tem apresentado melhoramentos significativos nas propriedades mecânicas e de barreira, o que tem sido atribuído a fraca adesão interfacial polímero/argila ${ }^{[20]}$. Além disso, nestes estudos, as argilas organofílicas empregadas são geralmente produtos comerciais, tais como Cloisites e Nanomers ${ }^{\circledR}$ fornecidas pela Southern Clay Company e Nanocor, respectivamente ${ }^{[24-32]}$, que são de custo elevado. O uso de argilas organofílicas desenvolvidas com tecnologia nacional para preparação de filmes de nanocompósitos a base de PP poderá promover o desenvolvimento do mercado interno e possibilitar a produção de nanocompósitos mais competitivos no mercado.

Visando, aprimorar a adesão interfacial entre PP/argila organofílica e melhor aproveitamento dos insumos nacionais, neste

Autor para correspondência: Suédina M. de L. Silva, Unidade Acadêmica de Engenharia de Materiais, Universidade Federal de Campina Grande - UFCG, Rua Aprígio Veloso, 882, Bodocongó, CEP 58429-900, Campina Grande, PB, Brasil, e-mail: suedina@dema.ufcg.edu.br 
estudo foi avaliada a influência do tipo e do teor de compatibilizante e do tipo de resina, na morfologia, propriedades mecânicas e de barreira ao vapor d'água de filmes de nanocompósitos PP/argila organofílica, preparados com tecnologia nacional, para aplicação no setor de embalagens.

\section{Experimental}

\section{Materiais}

Dois tipos de polipropileno foram empregados na preparação dos filmes de nanocompósitos. PP H401 (índice de fluidez de $7,5 \mathrm{~g} / 10 \mathrm{~min}$ a $230{ }^{\circ} \mathrm{C} / 2,16 \mathrm{~kg}$ - ASTM D 1238) e PP H07D-00 (índice de fluidez de $7,3 \mathrm{~g} / 10 \mathrm{~min}$ a $230^{\circ} \mathrm{C} / 2,16 \mathrm{~kg}$ - ASTM D 1238), produzidos pela Braskem/Brasil e pela INEOS - Olefins \& Polymers USA, respectivamente. Esses polipropilenos (PPs) são indicados para aplicação em embalagens flexíveis e de acordo com os fabricantes as propriedades destes polímeros são semelhantes exceto pela resistência ao impacto Izod (Tabela 1).

Polipropileno modificado com anidrido maleico (PP-g-AM), com $1 \%$ em massa de anidrido maleico, de nome comercial Polybond®3200 (índice de fluidez de $115 \mathrm{~g} / 10 \mathrm{~min}$ a $190{ }^{\circ} \mathrm{C} / 2,16 \mathrm{~kg}$ e temperatura de fusão de $160-170{ }^{\circ} \mathrm{C}$ ) e o copolímero de etileno e álcool vinílico $(E V O H)$ de nome comercial Soarnol ET3803 (índice de fluidez de $3,2 \mathrm{~g} / 10 \mathrm{~min}$ a $210{ }^{\circ} \mathrm{C} / 2,16 \mathrm{~kg}$ e temperatura de fusão de $173{ }^{\circ} \mathrm{C}$ ), fornecidos pela Chemtura Indústria Química do Brasil Ltda e Soarus LLC, respectivamente, foram usados na compatibilização dos nanocompósitos de PP/argila organofílica.

A bentonita Argel 35 (AN), silicato em camadas formado por duas folhas tetraédricas (T) a base de silício-oxogênio intercaladas com uma folha octaédrica (O), constituída fundamentalmente, de alumínio e ou magnésio-hidroxilas, com capacidade de troca catiônica (CTC) de 0,92 meq. $\mathrm{g}^{-1}$ de argila, conforme determinada previamente $^{[33]}$, foi fornecida pela Bentonit União Nordeste (BUN-Campina Grande/Brasil). Esta argila foi purificada, visando a remoção de matéria orgânica, de acordo com procedimento reportado por Araújo et al. (2007) $)^{[34]}$. O surfactante brometo de cetil trimetil amônio, $\mathrm{C}_{16} \mathrm{H}_{33}\left(\mathrm{CH}_{3}\right)_{3} \mathrm{~N}^{+} \mathrm{Br}$, fornecido pela Vetec, foi usado como recebido na modificação orgânica da argila purificada. A argila purificada modificada organicamente com tecnologia naciona ${ }^{[18]}$ foi codificada como APO.

\section{Preparação das amostras}

A preparação das amostras foi realizada em três etapas. $\mathrm{Na}$ primeira, visando avaliar o efeito do tipo de compatibilizante (PP-g-AM e EVOH), concentrados de compatibilizante e argila organofílica APO, na proporção 3:1 em massa, foram preparados em um misturador interno Haake Rheomix 600, operando com rotores do tipo roller a $170{ }^{\circ} \mathrm{C}$ e $60 \mathrm{rpm}$ por 12 minutos. Depois de triturados e secos em estufa a vácuo $\left(80{ }^{\circ} \mathrm{C} / 24\right.$ horas), o material foi diluído no PP H401 (P4) para a obtenção de filmes de nanocompósitos via intercalação no estado fundido empregando extrusora de filme plano da AX Plásticos. As zonas de aquecimento foram estabelecidas num gradiente de 180 a $190{ }^{\circ} \mathrm{C}$, com um torque de $42 \mathrm{~N} / \mathrm{m}$ e velocidade de rotação da rosca de $90 \mathrm{rpm}$. Os filmes obtidos com concentrações de $1 \%$ em massa de argila e $15 \%$ dos compatibilizantes PP-g-AM e EVOH foram codificados como P415AMAPO e P415OHAPO, respectivamente.

Na segunda etapa foi avaliado o efeito do teor do compatibilizante PP-g-AM na morfologia dos nanocompósitos PP H401/argila organofílica, onde foram testados três teores $(15,24$ e $34 \%$ em massa) do agente compatibilizante PP-g-AM. A escolha de 15\%, como o menor teor a ser testado, foi feita com base em estudo reportado na literatura ${ }^{[1]}$ onde os autores avaliaram o efeito de 5 teores de PP-g-AM (contendo o mesmo teor de anidrido maleico do PP-g-AM empregado no presente estudo - $1 \%$ em massa) no sistema PP/argila organofílica Cloisite 20A e mostraram que o teor de $15 \%$ em massa foi a quantidade mínima para garantir a dispersão da argila no polímero, em escala nanométrica.

Concentrados de PP-g-AM e argila organofílica APO nas proporções de 3:1, 5:1 e 7:1 em massa foram preparados em um misturador interno Haake Rheomix 600, operando sob as mesmas condições acima descritas. Depois de triturados e secos em estufa a vácuo $\left(80^{\circ} \mathrm{C} / 24\right.$ horas), foram misturados com o PP H401 (P4) e com uma quantidade de PP-g-AM suficiente para garantir teores de 15,24 e $34 \%$ de compatibilizante e $1 \%$ de argila organofílica APO nos filmes dos nanocompósitos obtidos. Os filmes contendo $1 \%$ de argila organofílica e 15, 24 e 34\% de PP-g-AM foram codificados como P415AMAPO, P424AMAPO e P434AMAPO.

$\mathrm{Na}$ terceira etapa foi avaliada a influência do tipo de resina na morfologia, propriedades mecânicas e de barreira dos filmes. Nessa fase, nanocompósitos com PP H401 (P4) e PP H07D-00 (P7) foram preparados com 1\% de argila organofílica APO e 15\% de PP-g-AM. Estes nanocompósitos foram codificados como P415AMAPO P715AMAPO, respectivamente. A metodologia empregada para preparação destes nanocompósitos foi a mesma descrita na primeira etapa.

\section{Caracterização}

As medidas de difração de raio $\mathrm{X}$ (DRX) foram conduzidas a temperatura ambiente em equipamento Shimadzu XDR-6000 operando a $40 \mathrm{kV}$ e $30 \mathrm{~mA}$ e com um comprimento de onda $\lambda \mathrm{CuK} \alpha=1,5406 \AA$. As amostras foram examinadas em um intervalo de $2 \theta$ entre 2 e $8^{\circ}$, a uma taxa de varredura de $2 \%$ min. Essa técnica é empregada para verificar a intercalação das cadeias poliméricas nas galerias da argila através da sua expansão interplanar basal e portanto, investigar a morfologia dos nanocompósitos obtidos ${ }^{[6,10,35,36]}$.

Os ensaios de resistência à tração (ASTM D 882), resistência à perfuração (ASTM F1306) e resistência da termossoldagem à tração (ASTM D 882) foram conduzidos a temperatura de $23{ }^{\circ} \mathrm{C}$ em máquina universal de ensaios Emic (DL500), operando a uma velocidade de $50 \mathrm{~mm} / \mathrm{min}$ empregando filmes com espessura na faixa de 60 a $100 \mu \mathrm{m}$.

No ensaio de resistência à tração o fator de ruptura (expresso em $\mathrm{N} / \mathrm{mm}$ ), relação entre a força máxima de tração por unidade de largura inicial do corpo de prova, parâmetro alternativo para

Tabela 1. Propriedades típicas dos polipropilenos PP H401 e PP H 07D-00.

\begin{tabular}{lccc}
\multicolumn{1}{c}{ Propriedade } & Método ASTM & Unidade & Valores \\
\cline { 3 - 4 } & & $\mathrm{MPa}$ & PP H401 (P4) \\
Módulo de Flexão a $23{ }^{\circ} \mathrm{C}$ & $\mathrm{D} 790$ & $\mathrm{MPa}$ & 1400 \\
Resistência à tração no escoamento a $23{ }^{\circ} \mathrm{C}$ & $\mathrm{D} 638$ & 33 & 37 \\
Temperatura de deflexão térmica a $0,455 \mathrm{MPa}$ & $\mathrm{D} 648$ & $\mathrm{C}$ & 92 \\
Resistência ao Impacto Izod a $23{ }^{\circ} \mathrm{C}$ & $\mathrm{D} 256$ & $\mathrm{~J} / \mathrm{m}$ & 32 \\
\hline
\end{tabular}

Fonte: http://www.braskem.com.br e http://www.ineos-op.com. 
caracterizar a resistência à tração de filmes cuja seção transversal não é homogênea, a exemplo dos materiais multicamadas, ou cuja espessura não pode ser determinada com a precisão necessária ${ }^{[37]}$, foi determinado empregando filmes com $25 \mathrm{~cm}$ de comprimento e $5 \mathrm{~cm}$ de largura.

Na determinação da resistência à perfuração (expressa em N) foram empregadas amostras de filmes com diâmetro de $12 \mathrm{~cm}$. Este teste é comumente aplicado na caracterização de filmes poliméricos, utilizados no acondicionamento de produtos, que podem estar em contato com pontas, cantos vivos e outros tipos de protuberâncias capazes de danificar a embalagem durante o acondicionamento e a distribuição ${ }^{[7]}$.

Para o ensaio da termossoldagem à tração, filmes de $25 \mathrm{~cm}$ de comprimento e $5 \mathrm{~cm}$ de largura foram selados, segundo norma ASTM D F88-00, empregando temperatura média de $165{ }^{\circ} \mathrm{C}$ e pressão de $3,5 \mathrm{kgf.cm}^{-2}$ por 0,5 segundos. A termossoldagem, processo pelo qual duas estruturas contendo pelo menos uma camada de termoplástico na interface são unidas pela ação combinada de calor e pressão, é uma das mais importantes propriedades dos materiais utilizados em embalagens flexíveis e, fundamental para manter a integridade da embalagem ${ }^{[37]}$. O ensaio de resistência da termossoldagem à tração foi realizado na direção da máquina e é expresso em $\mathrm{N} / \mathrm{mm}$.

A taxa de permeabilidade ao vapor d'água (TPVA), expressa em g água/(m2.dia), foi determinada em equipamento TSY W3/3 da Labthink Instruments Co. LTD, segundo a norma ASTM E96-00 (temperatura de $38{ }^{\circ} \mathrm{C}$ e umidade relativa de $99 \%$ ). Os filmes com espessura na faixa de 60 a $100 \mu \mathrm{m}$ e diâmetro de $14 \mathrm{~cm}$ foram mantidos em dessecador por 24 horas antes da realização do teste.

\section{Resultados e Discussão}

Os padrões de difração de raio X da argila APO e dos filmes de PP $\mathrm{H} 401$, contendo $1 \%$ de argila purificada organofilizada APO, compatibilizados com $15 \%$ de EVOH e $15 \%$ de PP-g-AM (P415OHAPO e P415AMAPO) estão apresentados na Figura 1. De acordo com os resultados, fica evidenciado que a incorporação de $1 \%$ de argila APO aos PPs compatibilizados resultou na formação de nanocompósitos, conforme indicado pelo aumento da distância interplanar basal (001) da argila dispersa no polímero, e que o tipo de compatibilizante afetou a morfologia dos nanocompósitos obtidos. Nanocompósitos compatibilizados com EVOH (P415OHAPO) apresentam morfologia intercalada ordenada caracterizada pelo perfil do plano difratado (001) da argila, bem definido cuja abertura corresponde a 3,65 nm. Por outro lado, quando os nanocompósitos foram compatibilizados com PP-g-AM (P415AMAPO), a distância interplanar basal da argila (001) foi superior a 3,65 nm e uma banda é observada sugerindo que neste caso nanocompósitos com estrutura intercalada desordenada foram obtidos devido a maior penetração das cadeias poliméricas entre as camadas da argila ${ }^{[15,17,38]}$.

Embora nanocompósitos tenham sido obtidos quando os sistemas $\mathrm{PP} /$ argila organofílica APO foram compatibilizados com EVOH e PP-g-AM, o último mostrou um melhor efeito compatibilizante devido à maior abertura do plano basal (intercalação) juntamente com a quebra parcial da estrutura cristalina (esfoliação parcial) da argila. A maior compatibilidade do PP-g-AM com o PP e as fortes interações entre PP-g-AM e a argila (Figura 2) podem ter resultado em uma melhor intercalação, da argila por polímero, no estado fundido. Considerando que o EVOH não é totalmente compatível com o PP e que o tipo de ligação entre EVOH e argila é mais fraca (Figura 3) do que aquela formada entre PP-g-AM e a argila, acreditase que as interações PP-argila nos nanocompósitos compatibilizados com EVOH devem ter sido mais fracas e desta forma, a intercalação do polímero na argila tenha sido menos favorecida neste caso.
Para avaliar o efeito do teor de compatibilizante na morfologia dos nanocompósitos PP/argila APO, elegeu-se apenas o sistema contendo PP-g-AM já que nanocompósitos com estrutura intercalada desordenada (Figura 1) só foram obtidos quando este agente compatibilizante foi utilizado.

Os padrões de raio $\mathrm{X}$ dos filmes dos nanocompósitos contendo $1 \%$ em massa de argila APO e compatibilizados com 15, 24 e $34 \%$ em massa de PP-g-AM, codificados como P415AMAPO, P424AMAPO e P434AMAPO, respectivamente, estão apresentados na Figura 4. Observa-se que os padrões de DRX dos três nanocompósitos estudados são similares. Em todos os casos os valores de $2 \theta$ foram deslocados à esquerda, máximos das reflexões inferiores a 2 graus, denotando picos em (001) alargados indicando que as camadas de argila estão dispersas de forma desordenada na matriz polimérica. Além disso, a diminuição na intensidade dos picos (001) pode ser resultado de uma esfoliação parcial das camadas da argila[11]. Com base nestes resultados, acredita-se que o máximo grau de intercalação, nas condições de fusão empregadas para preparação dos filmes dos nanocompósitos de PP contendo $1 \%$ em massa de argila organofílica APO, tenha sido atingido quando $15 \%$ em massa de PP-g-AM foi usado. É possível que este teor de compatibilizante tenha sido suficiente para promover a completa reação entre os grupos hidroxílicos da camada octaédrica $(\mathrm{O})$ da argila APO e o anidrido maleico do PP-g-AM (Figura 2). Sendo assim, o teor de $15 \%$ de PP-g-AM foi o escolhido para preparar os nanocompósitos com PP H07D-00 (P715AMAPO) e comparar as suas propriedades mecânicas e de barreira com as dos nanocompósitos preparados com PP H401 (P415AMAPO). Desta forma pode-se avaliar o efeito do tipo de matriz nas referidas propriedades.

Conforme mostrado na Figura 5, os padrões de difração de raio $\mathrm{X}$ dos nanocompósitos preparados com as matrizes poliméricas PP H401 e PP H07D-00 (P415AMAPO e P715AMAPO) são semelhantes. O nanocompósito P715AMAPO também apresentou pico de difração (001) de baixa intensidade e alargado (formação de banda), o qual foi deslocado para valores de $2 \theta$ menores do que o da argila APO e inferiores a $2^{\circ}$, indicando que as camadas de argila devem estar dispersas de forma desordenada na matriz PP H07D-00, praticamente da mesma forma que na matriz PP H401. Entretanto, conforme evidenciado nas Figuras 6-9, as propriedades mecânicas e de barreira ao vapor d'água dos nanocompósitos preparados com as matrizes poliméricas PP H401 e PP H07D-00 (P415AMAPO e P715AMAPO) são diferentes.

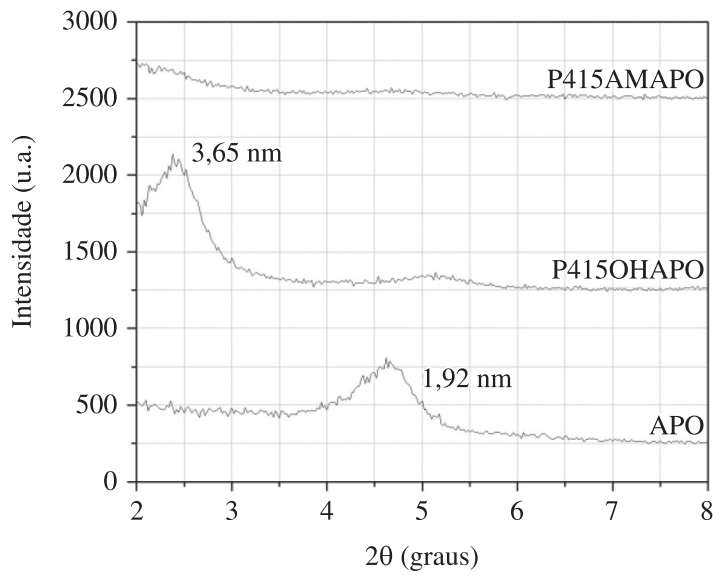

Figura 1. Padrões de difração de raio $X$ da argila organofílica APO e dos filmes dos nanocompósitos, contendo $1 \%$ de APO, compatibilizados com EVOH (P415OHAPO) e PP-g-AM (P415AMAPO). 


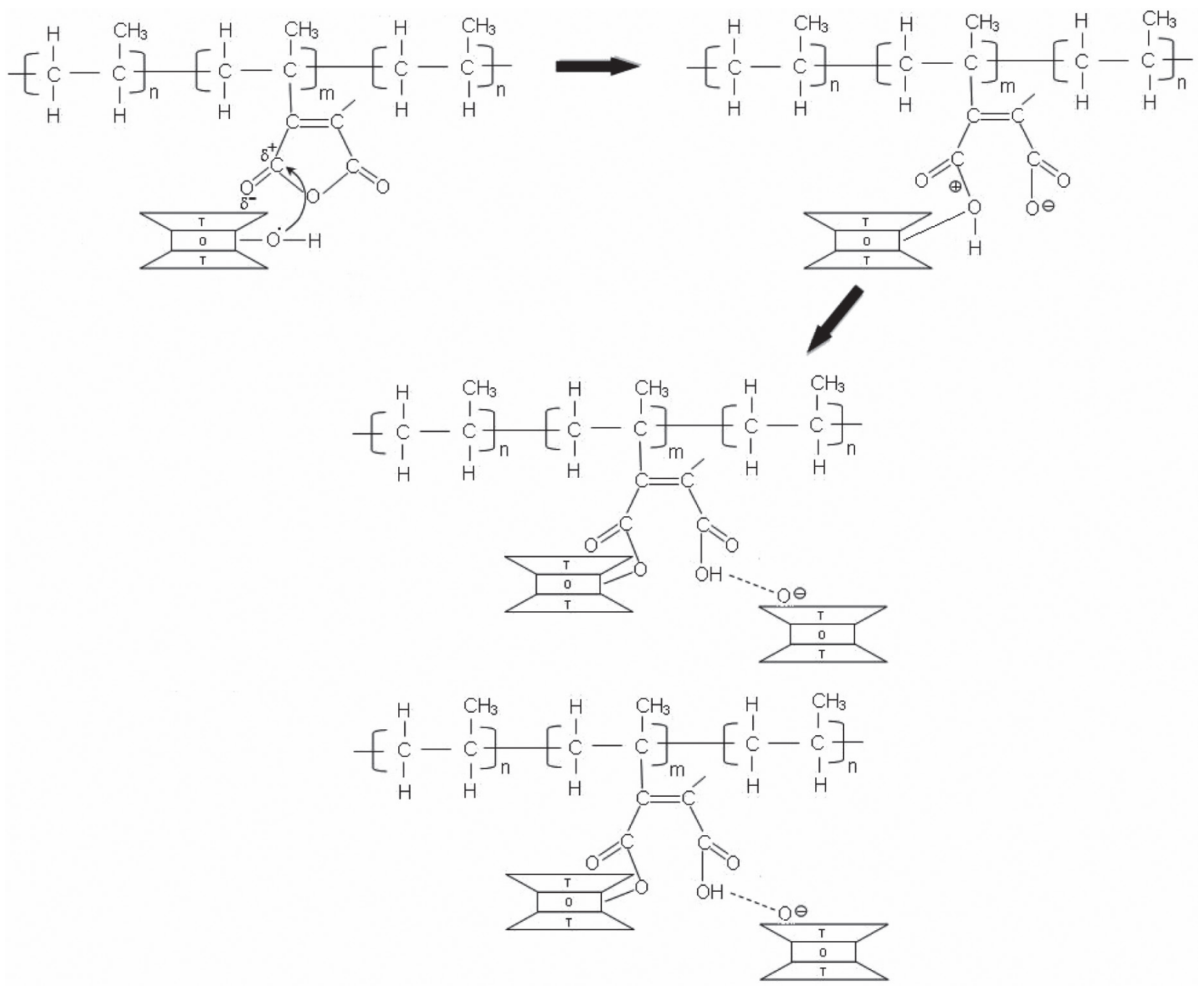

Figura 2. Mecanismo proposto para a reação entre os grupos OH da folha octaédrica (O) da argila e anidrido maléico do PP-g-AM. Adaptado de Dumont et al. ${ }^{383}$.
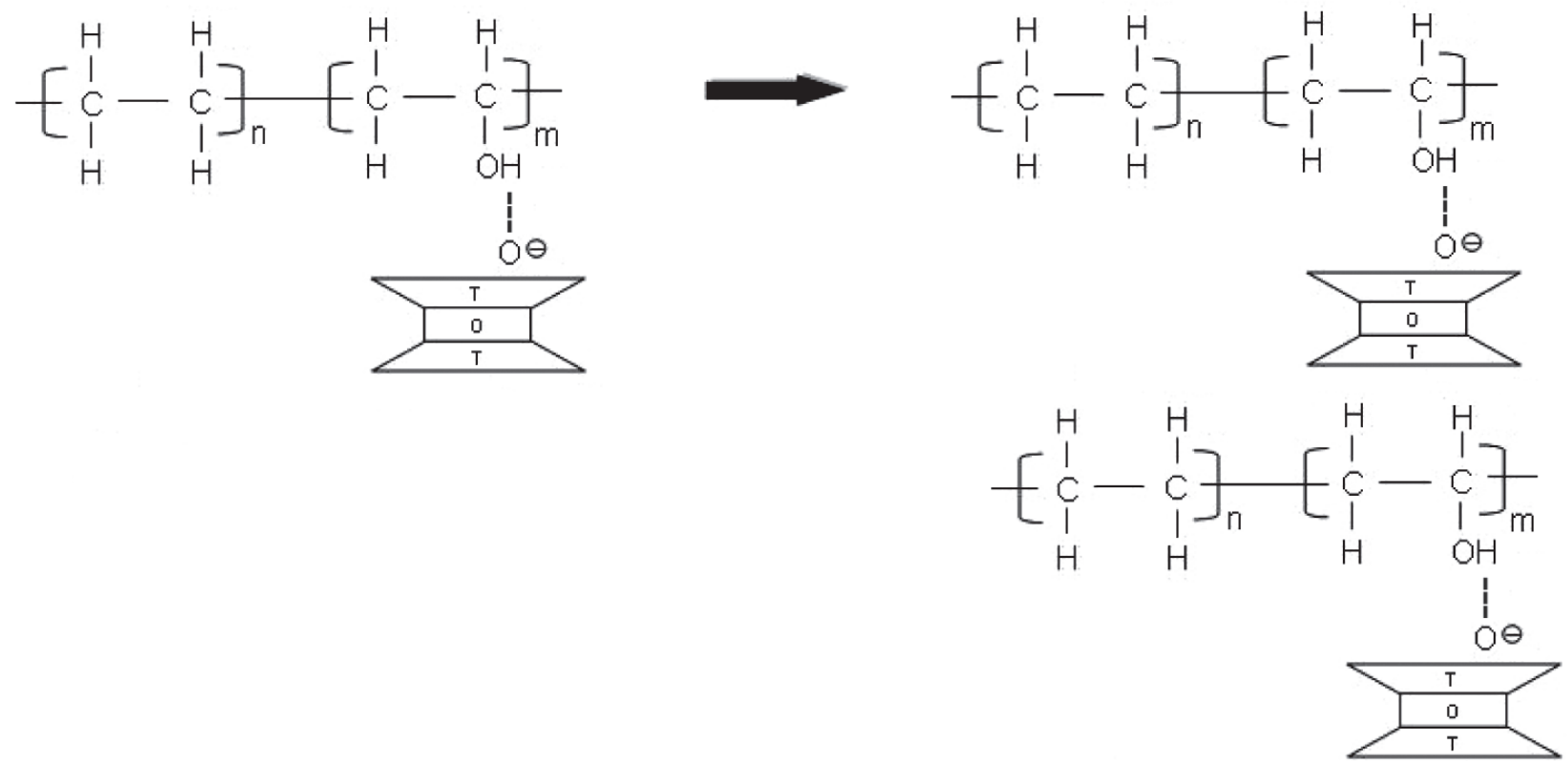

Figura 3. Mecanismo proposto para a reação entre os oxigênios da folha tetraédrica (T) da argila e hidroxila do EVOH. 


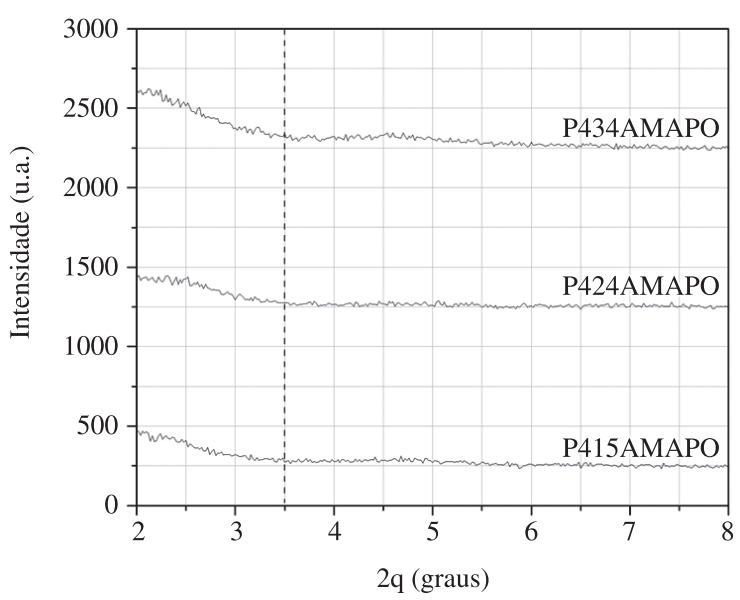

Figura 4. Padrões de difração de raio $X$ dos filmes dos nanocompósitos de PP H401, contendo 1\% de APO, compatibilizados com 15, 24 e $34 \%$ de PP-g-AM, P415AMAPO, P424AMAPO e P434AMAPO, respectivamente.

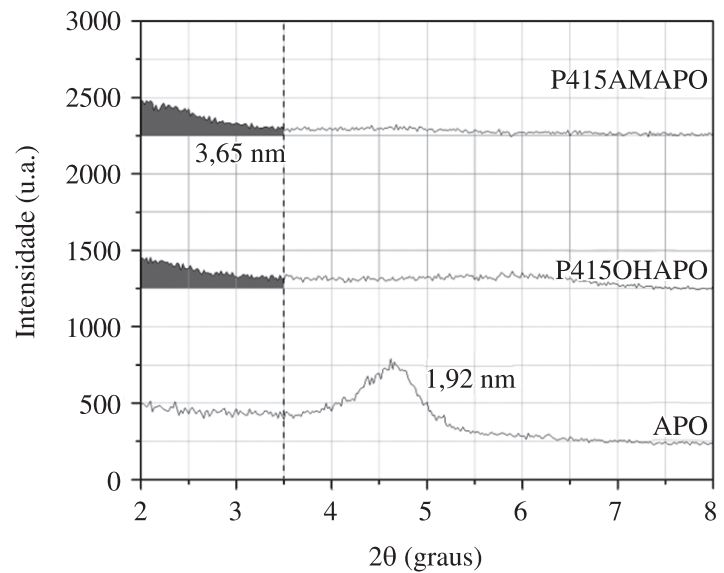

Figura 5. Padrões de difração de raio $X$ da argila $\mathrm{APO}$ e dos nanocompósitos de PP H07D-00 e PP H401, contendo 1\% de APO, compatibilizados com 15\% de PP-g-AM, P715AMAPO e P4154AMAPO, respectivamente.

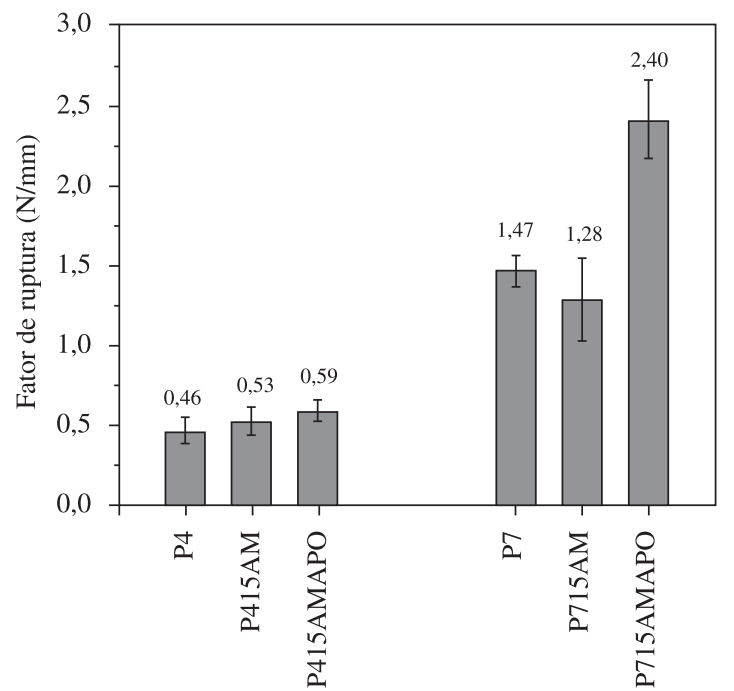

Figura 6. Fator de ruptura dos filmes dos PPs puros (P4 e P7), dos PPs/ PP-g-AM (P415AM e P715AM) e dos filmes dos nanocompósitos compatibilizados com 15\% de PP-g-AM e contendo $1 \%$ de argila APO (P415AMAPO e P715AMAPO).

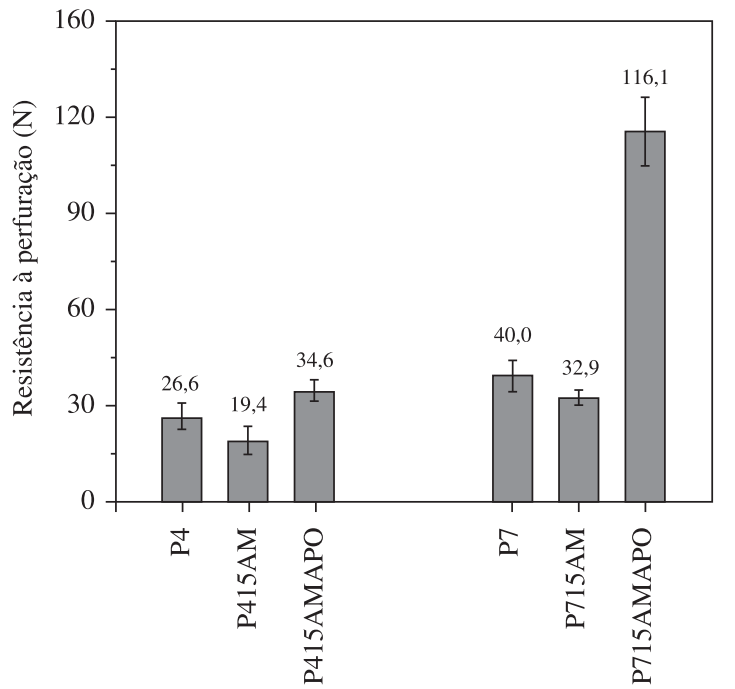

Figura 7. Resistência à perfuração dos filmes dos PPs puros (P4 e P7), dos PPs/PP-g-AM (P415AM e P715AM) e dos filmes dos nanocompósitos compatibilizados com $15 \%$ de PP-g-AM e contendo $1 \%$ de argila APO (P415AMAPO e P715AMAPO).

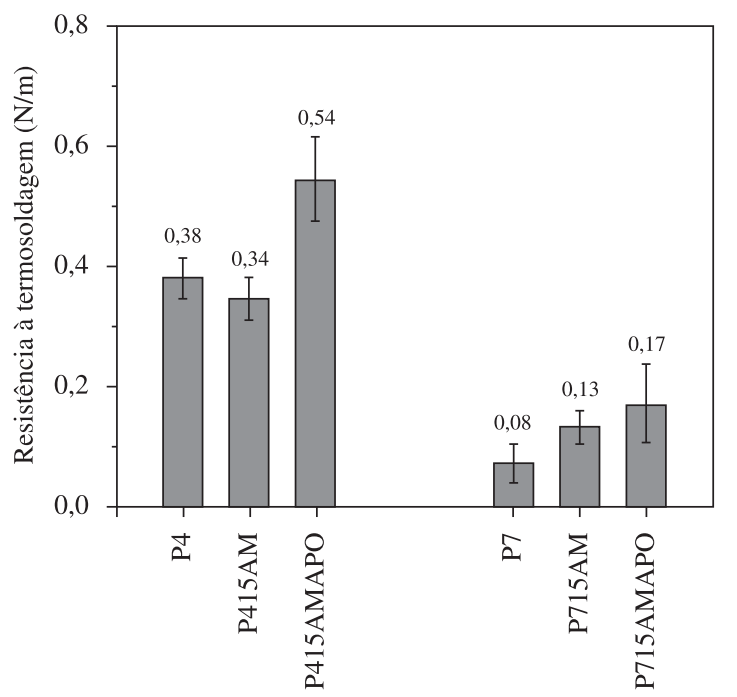

Figura 8. Resistência da termossoldagem à tração dos filmes dos PPs puros, dos PPs/PP-g-AM e dos filmes dos nanocompósitos PP H401 (P415AMAPO) e PP07D (P715AMAPO) compatibilizados com PP-g-AM contendo $1 \%$ de argila APO.

De acordo com as Figuras 6-8, os filmes dos nanocompósitos preparados com PP H401 e PP H07D-00, compatibilizados com $15 \%$ de PP-g-AM e contendo 1\% de argila APO (P415AMAPO e P715AMAPO), apresentaram maiores valores de fator de ruptura (Figura 6), resistência à perfuração (Figura 7) e resistência à termossoldagem (Figura 8) do que os filmes de PP H401 e PP H07D-00 misturados com 15\% de PP-g-AM (P415AM e P715AM) e do que os filmes de PP H401 e PP H07D-00 puros (P4 e P7). Comparando os valores apresentados pelos filmes dos nanocompósitos P415AMAPO e P715AMAPO com os apresentados pelos filmes dos PPs misturados com PP-g-AM (P415AM e P715AM), os aumentos foram, respectivamente, de aproximadamente 11 e $87 \%$ no fator de ruptura (Figura 6); de 78 e $252 \%$ na resistência à perfuração (Figura 7) e de 58 e $30 \%$ na resistência a termossoldagem (Figura 8). Se comparado com os filmes dos PPs puros (P4 e P7) estes aumentos foram, respectivamente, de aproximadamente 28 e $63 \%$ no fator de ruptura 
(Figura 6); de 30 e 190\% na resistência à perfuração (Figura 7) e de 42 e $112 \%$ na resistência a termossoldagem (Figura 8). Os aumentos das propriedades mecânicas dos filmes dos nanocompósitos, comparados aos dos filmes dos polímeros não carregados, podem estar associados às fortes interações polímero-argila que permitem a efetiva transferência das tensões da matriz para a carga e com a alta pureza da argila organofílica APO preparada com tecnologia nacional, por nosso grupo de pesquisa, que na ausência de impurezas micrométricas, que agem como concentradores de tensão, suportam maiores esforços mecânicos.

As Figuras 6-8 mostram também que, embora o PP H401 (P4) e o PP H07D-00 (P7) sejam semelhantes, de acordo com as informações fornecidas pelos seus respectivos fabricantes (Tabela 1), as pequenas diferenças nas propriedades da matriz polimérica afetaram consideravelmente os valores das propriedades mecânicas dos filmes dos nanocompósitos obtidos. Os aumentos nas propriedades mecânicas dos filmes dos nanocompósitos preparados com o PP H07D-00 (P7) foram maiores do que os daqueles preparados com PP H401 (P4). De acordo com Gianelli et al. ${ }^{[39]}$, características da resina base tais como massa molar e distribuição de massa molar, também influenciam as propriedades dos nanocompósitos obtidos uma vez que modificam a cinética de intercalação da argila pelas cadeias poliméricas e afetam a viscosidade do fundido, alterando o nível de cisalhamento sofrido pelo polímero no estado fundido e, desta forma, a interação polímero-argila. Obviamente, quando a interação polímero-argila é melhorada, as tensões são muito mais efetivamente transferidas da matriz polimérica para a carga inorgânica e, portanto, um aumento maior nas propriedades mecânicas é esperado. Assim, a interação polímero-argila parece ser o fator determinante nas propriedades mecânicas dos nanocompósitos preparados com as diferentes matrizes poliméricas, uma vez que os nanocompósitos P415AMAPO e P715AMAPO apresentaram níveis de intercalação da argila pelos polímeros semelhantes, conforme Figura 5.

A Figura 9 apresenta os valores da taxa de permeação ao vapor d'água (TPVA) dos PPs puros (P4 e P7), dos PPs misturados com $15 \%$ de PP-g-AM (P415AM e P715AM) e dos nanocompósitos preparados com P4 e P7, compatibilizados com 15\% de PP-g-AM e contendo $1 \%$ de argila APO (P415AMAPO e P715AMAPO). Pode-se observar que os filmes dos nanocompósitos preparados com PP H 401 (P415AMAPO) apresentaram valores da TPVA semelhantes aos dos filmes P415AM e P4. Por outro lado, os filmes dos nanocompósitos preparados com PP H 07D-00 (P715AMAPO) apresentaram menores valores da TPVA do que os filmes P715AM; mas quando comparado com o PP H07D-00 puro (P7) estes valores foram superiores.

Apesar da boa dispersão, intercalação e esfoliação parcial da argila APO nas matrizes de PP, a taxa de permeação ao vapor d'água no nanocompósito P415AMAPO foi semelhante ao da matriz P415AM e no nanocompósito P715AMAPO foi cerca de $17 \%$ menor do que o da matriz P715AM. Se por um lado o coeficiente de difusão diminui com a presença do compatibilizante (melhor dispersão da argila), por outro, a solubilidade aumenta (forte interação entre grupos polares do compatibilizante e a água), efeitos que somados determinam a permeabilidade final das amostras. Sendo esta a possível razão para a permeabilidade ao vapor d'água no nanocompósito PP715AMAPO ter tido uma redução de apenas $17 \%$ em relação à matriz polimérica.

Em resumo, os nanocompósitos preparados com a matriz polimérica P715AM (P715AMAPO) apresentaram melhores propriedades mecânicas, excetuando a resistência da termossoldagem à tração, do que os preparados com a matriz polimérica $\mathrm{P} 415 \mathrm{AM}$ (P415AMAPO). A transparência destes nanocompósitos (P715AMAPO) foi semelhante à da matriz polimérica sem argila

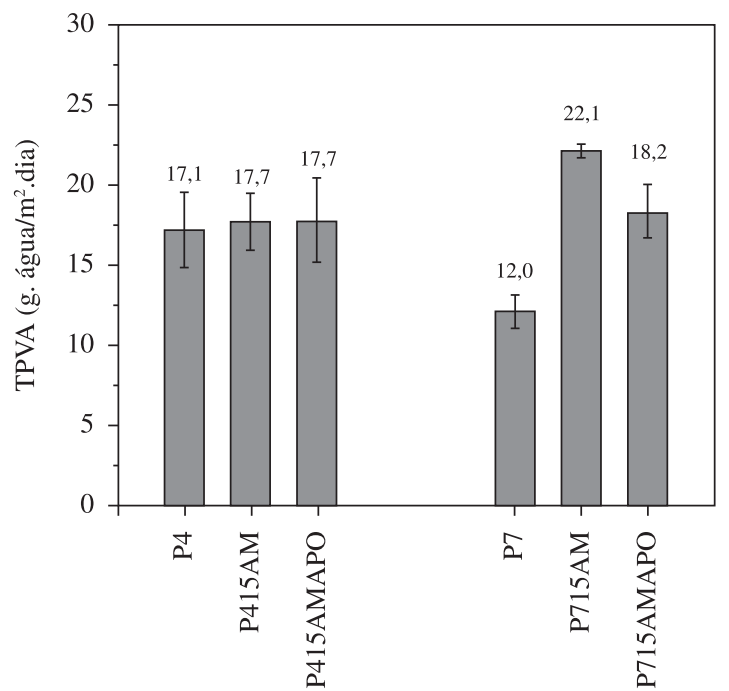

Figura 9. Barreira ao vapor d'água dos filmes dos PPs puros (P4 e P7), dos PPs misturados com 15\% de PP-g-AM (P415AM e P715AM) e dos nanocompósitos preparados com P4 e P7, compatibilizados com 15\% de PP-g-AM e contendo 1\% de argila APO (P415AMAPO e P715AMAPO).
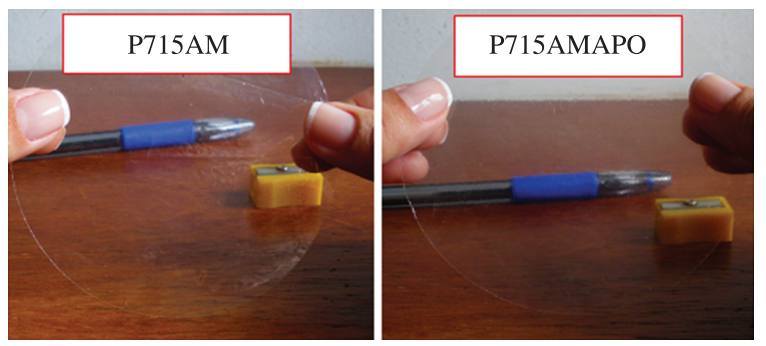

Figura 10. Fotografias dos filmes da mistura de PPH70D-00 com 15\% de PP-g-AM (P715AM) e dos nanocompósitos preparados com P715AM e contendo $1 \%$ de argila APO (P715AMAPO).

(P715AM) (Figura 10). Apesar da taxa de permeabilidade ao vapor d'água (TPVA) dos nanocompósitos preparados com a matriz P715AM (P715AMAPO) ter sido semelhante à dos preparados com a matriz P415AM (P415AMAPO), a incorporação de 1\% em massa da argila organofílica APO à matriz P715AM resultou em pequena redução (17\% em relação à matriz) da TPVA deste nanocompósito enquanto nenhuma alteração nesta propriedade foi observada quando a argila foi incorporada à matriz PP415AM.

\section{Conclusões}

Filmes de nanocompósitos PP/argila contendo $1 \%$ em massa de argila organofílica foram preparados por extrusão plana. Filmes foram produzidos com duas matrizes de PP (PP H401 e PP H07D-00) utilizando polipropileno enxertado com anidrido maleico (PP-g-AM) e copolímero de etileno e álcool vinílico $(\mathrm{EVOH})$ como agentes compatibilizantes. Ficou evidenciado que a morfologia dos nanocompósitos foi afetada pelo tipo de compatibilizante empregado e que aqueles compatibilizados com EVOH apresentaram morfologia intercalada ordenada enquanto uma estrutura intercalada desordenada tendendo a esfoliada foi obtida para os compatibilizados com PP-g-AM. Nas condições experimentais adotadas e nos teores de PP-g-AM utilizados (15-34\%), a adição de $15 \%$ em massa de PP-g-AM foi suficiente para promover o máximo grau de intercalação da argila pelo polímero. Dados de DRX sugerem que as morfologias e as propriedades de barreira ao vapor d'água dos nanocompósitos preparados com os polipropilenos PP H401 e PP H07D-00 são 
semelhantes. Entretanto, aqueles preparados com PP H07D-00 apresentaram melhores propriedades mecânicas, excetuando a resistência da termossoldagem à tração, do que os preparados com a matriz polimérica PP H401 e a transparência dos nanocompósitos preparados com PP H07D-00 foi semelhante à da matriz polimérica sem argila.

\section{Agradecimentos}

Os autores agradecem a CAPES e ao CNPq, pelo apoio financeiro, à Felinto pela doação dos polímeros (PPs), e determinação das propriedades mecânicas e de barreira, à Chemtura e Soarus pela doação dos compatibilizantes e a Bentonit União Nordeste S/A pela doação da bentonita.

\section{Referências Bibliográficas}

1. Moore, G. - "Nanotecnologia em Embalagens", Editora Blucher, São Paulo (2010).

2. Chaudhry, Q.; Scotter, M.; Blackburn, J.; Ross, B.; Boxall, A.; Castle, L.; Aitken, R. \& Waltkins, R. - Addit. Contam.: Part A, 25, p.241 (2008). http://dx.doi.org/10.1080/02652030701744538

3. Bradley, E. L.; Castle, L. \& Chaudhry, Q. - Trends Food Sci. Tech., 22, p.604 (2011). PMid:21299575. http://dx.doi.org/10.1016/j. tifs.2011.01.002

4. Kawasumi, M.; Haseagawa, N.; Kato, M.; Usuki, A. \& Okada, A. - Macromolecules, 30, p.6333 (1997). http://dx.doi.org/10.1021/ ma961786h

5. Manias, E.; Touny, A.; Wu, L.; Strawhecker, K.; Lu, B., \& Chung, T. C. - Chem. Mater., 13, p.3516 (2001). http://dx.doi.org/10.1021/ $\mathrm{cm} 0110627$

6. Ray, S. S. \& Okamoto, M. - Prog. Polym. Sci., 28, p.1539 (2003).

7. García-López, D.; Picazo, O.; Merino, J. C.; Pastor, J. M. - Eur. Polym. J. 39, p.945 (2003).

8. Tidjani, A.; Wald, O.; Pohl, M.; Hentschel, M. P. \& Schartel, B. - Polym. Degrad. Stab., 82, p.133 (2003). http://dx.doi.org/10.1016/ S0141-3910(03)00174-5

9. Xu, W.; Liang, G.; Zhai, H.; Tang, S.; Hang, G. \& Pan, E. - Eur. Polym. J., 39, p.1467 (2003). http://dx.doi.org/10.1016/S00143057(03)00015-6

10. Utracki, L. A. - "Clay-Containing Polymeric Nanocomposites", Rapra Technology Limited, United Kingdom (2004).

11. Lertwilmolnun, W. \& Vergnes, B. - Polymer, 46, p.3462 (2005). http:// dx.doi.org/10.1016/j.polymer.2005.02.018

12. Ramos Filho, F. G.; Mélo, T. J. A.; Rabello, M. S. \& Silva, S. M. L. S. - Polym. Degrad. Stab., 89, p.383 (2005). http://dx.doi.org/10.1016/j. polymdegradstab.2004.12.011

13. López-Quintanilla, M. L.; Sánchez-Valdés, S.; Ramos de Valle, L. F. \& Miranda, R. G. - Polym. Bull., 57, p.385 (2006).

14. Lopez-Quintanilla, M. L.; Sánchez-Valdés, S.; Ramos de Valle, L. F. \& Medellin-Rodriguez, F. J. - J. Appl. Polym. Sci., 100, p.4748 (2005). http://dx.doi.org/10.1002/app.23262

15. Kim, D. H.; Fasulo, P. D.; William, R. R. \& Donald, R. P. - Polymer., 48, p.5308 (2007). http://dx.doi.org/10.1016/j.polymer.2007.07.011

16. Rohlmann, C. O.; Horst, M. F.; Quinzani, L. M. \& Failla, M. D. - Eur. Polym. J., 44, p.2749 (2008). http://dx.doi.org/10.1016/j. eurpolymj.2008.07.006

17. Pascual J.; Fages, E.; Fenollar, O.; García, D. \& Balart, R. - Polym. Bull., 62, p.367 (2009). http://dx.doi.org/10.1007/s00289-008-0018-7

18. Silva, S. M. L.; Araujo, P. E. R.; Ferreira, K. R. M.; Canedo, E. L.; Carvalho, L. H. \& Raposo,C. M. O. - Polym. Eng. Sci., 49, p.1696 (2009). http://dx.doi.org/10.1002/pen.21399
19. Xie, S.; Harkin-Jones, E.; Shen, Y; Hornsby, P.; McAfee, M.; McNally, T.; Patel, R.; Benkreira, H. \& Coates, P. - Mater. Lett., 64, p.185 (2010). http://dx.doi.org/10.1016/j.matlet.2009.10.042

20. Morelli, F. C. \& Ruvolo Filho, A. - Polímeros, 20, p.121 (2010).

21. Abreu, D. A. P.; Losada, P. P.; Ângulo, I. \& Cruz, J.M. - Europ. Polym. J., 43, p.2229 (2007).

22. Manikantan, M. R. \& Varadharaju, N. - Packag. Technol. Sci., 24 (2011). http://dx.doi.org/10.1002/pts.925

23. Sirousazar, M.; Yari, M.; Achachlouei, B. F.; Arsalani, J. \& Mansoori, Y. - Polymers, 27 (2007).

24. Cui, L.; Hunter, D. L.; Yoon, P. J. \& Paul, D. R. - Polymer, 49, p.3762 (2008). http://dx.doi.org/10.1016/j.polymer.2008.06.028

25. Abu-Zurayk, R.; Harkin-Jones, E.; McNally, T.; Menary, G.; Martin, P. \& Armstrong, C. Compos. Sci. Technol., 69, p.1644 (2009). http:// dx.doi.org/10.1016/j.compscitech.2009.03.014

26. Dal Castel, C.; Bianchi, O.; Oviedo, M. A. S.; Liberman, S.A.; Mauler, R. S. \& Oliveira, R. V. B. - Mater. Sci. Eng. C, 29, p.602 (2009). http:// dx.doi.org/10.1016/j.compositesa.2009.03.006

27. Lai, S.-M.; Chen, W.-C.\& Zhu, X. S. - Compos. part A, 40, p.754. (2009). http://dx.doi.org/10.1016/j.compositesa.2009.03.006

28. Santos, K. S.; Liberman, S. A.; Oviedo, M. A. S. \& Mauler, R. S. - Compos. part A, 40, p.1199 (2009). http://dx.doi.org/10.1016/j. compositesa.2009.05.009

29. Hedayati, A. \& Arefazar, A. - Polym. Test., 28, p.128 (2009). http:// dx.doi.org/10.1016/j.polymertesting.2008.10.007

30. Chinellato, A. C.; Vidotti, S. E.; Hub, G.-H. \& Pessan, L. A. - Compos. Sci. Technol., 70, p.458 (2010). http://dx.doi.org/10.1016/j. compscitech.2009.11.020

31. Rousseaux, D. D. J.; Sallem-Idrissi, N.; Baudouin, A.; Devaux, J.; Godard, P.; Marchand-Brynaert, J. \& Sclavons, M. - Polymer, 52, p.443 (2011). http://dx.doi.org/10.1016/j.polymer.2010.11.027

32. Rhodes, J.; Smith, C. \& Stec, A. A. - Polym. Degrad. Stab., 96, p.277 (2011). http://dx.doi.org/10.1016/j.polymdegradstab.2010.07.002

33. Leite, I. F.; Soares, A. P. S.; Carvalho, L. H.; Malta, O. M. L.; Raposo, C. M. O. \& Silva, S. M. L. - J. Thermal. Anal. Calorim., 100, p.563 (2010). http://dx.doi.org/10.1007/s10973-009-0265-3

34. Araujo, P. E. R.; Araújo, S. S.; Raposo, C. M. O.; Silva, S. M. L. - "Poly(ethylene terephthalate)/PET/layered silicate nanocomposites: effect of bentonite purification on morphology/ behaviour relationship", in: Proceedings of the $23^{\circ}$ Polymer Processing. Society Annual Meeting, p.1, Salvador - BA, jun (2007).

35. Alexandre, M. \& Dubois, P. - Mater. Sci. Eng., 28, p.1 (2000). http:// dx.doi.org/10.1016/S0927-796X(00)00012-7

36. Pavlidou, S. \& Papaspyrides, C.D. - Prog. Polym. Sci., 33, p.1119 (2008). http://dx.doi.org/10.1016/j.progpolymsci.2008.07.008

37. Sarantópoulos, C. I. G. L; iliveira, L. M.; Padula, M.; Coltro, L.; Alves, R. M. V.; Garcia, E.C. - "Embalagens Plásticas Flexíveis. Principais Polímeros e Avaliação de Propriedades", CETEA/ITAL, Campinas (2002).

38. Dumont, M.-J.; Reyna-Valencia, A.; Edmond, J.-P \& Bousmina M. - J. Appl. Polym. Sci., 103, p.618 (2007). http://dx.doi.org/10.1002/ app. 25253

39. Gianelli, W.; Ferrara, G.; Camino, G.; Pellegatti, G.; Rosenthal, J. \& Trombini, R.C. - Polymer, 46, p.7037 (2005). http://dx.doi. org/10.1016/j.polymer.2005.05.149

Enviado: 16/06/11 Reenviado: 12/08/11 Aceito: 23/08/11 